\title{
Cochrane
}

Cochrane Database of Systematic Reviews

\section{Cognitive-emotional interventions for breathlessness in adults with advanced diseases (Protocol)}

Bolzani A, Rolser SM, Kalies H, Maddocks M, Rehfuess E, Hutchinson A, Gysels M, Higginson IJ, Booth S, Bausewein C

Bolzani A, Rolser SM, Kalies H, Maddocks M, Rehfuess E, Hutchinson A, Gysels M, Higginson IJ, Booth S, Bausewein C. Cognitive-emotional interventions for breathlessness in adults with advanced diseases. Cochrane Database of Systematic Reviews 2017, Issue 6. Art. No.: CD012682.

DOI: 10.1002/14651858.CD012682.

www.cochranelibrary.com 
TABLE OF CONTENTS

HEADER . . . . . . . . . . . . . . . . . . . . . . . . . . . . . . . . . . . . 1

ABSTRACT . . . . . . . . . . . . . . . . . . . . . . . . . . . . . . . . . . . . . . . 1

BACKGROUND . . . . . . . . . . . . . . . . . . . . . . . . . . . . . . . . . . . . . . . . . . . . . .

Figure 1. . . . . . . . . . . . . . . . . . . . . . . . . . . . . . . . . . . . . . $\quad 2$

Figure 2. . . . . . . . . . . . . . . . . . . . . . . . . . . . . . . . . . . . . .

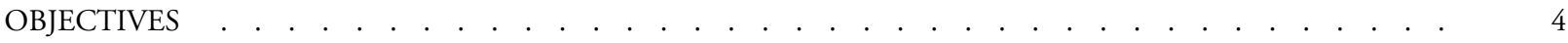

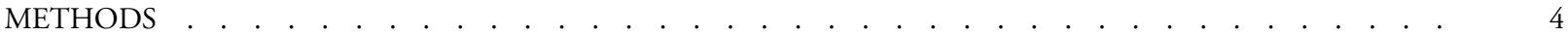

ACKNOWLEDGEMENTS . . . . . . . . . . . . . . . . . . . . . . . . . . . . . . . . . .

REFERENCES . . . . . . . . . . . . . . . . . . . . . . . . . . . . . . . . . . . . . . 9

APPENDICES . . . . . . . . . . . . . . . . . . . . . . . . . . . . . . . . . . . . . 11

CONTRIBUTIONS OF AUTHORS . . . . . . . . . . . . . . . . . . . . . . . . . . . . . . . . . . . . .

DECLARATIONS OF INTEREST . . . . . . . . . . . . . . . . . . . . . . . . . . . . . . . . . . . .

SOURCES OF SUPPORT . . . . . . . . . . . . . . . . . . . . . . . . . . . . . . . . . . . . . . . . . . . 


\title{
[Intervention Protocol]
}

\section{Cognitive-emotional interventions for breathlessness in adults with advanced diseases}

\author{
Anna Bolzani ${ }^{1}$, Stefanie M Rolser ${ }^{1}$, Helen Kalies ${ }^{1}$, Matthew Maddocks ${ }^{2}$, Eva Rehfuess ${ }^{3}$, Ann Hutchinson ${ }^{4}$, Marjolein Gysels ${ }^{5}$, Irene \\ J Higginson $^{6}$, Sara Booth ${ }^{7}$, Claudia Bausewein ${ }^{1}$ \\ ${ }^{1}$ Department of Palliative Medicine, Munich University Hospital, LMU Munich, Munich, Germany. ${ }^{2}$ Department of Palliative Care, \\ Policy and Rehabilitation, Cicely Saunders Institute, King's College London, London, UK. ${ }^{3}$ Institute for Medical Informatics, Biometry \\ and Epidemiology, Pettenkofer School of Public Health, LMU Munich, Munich, Germany. ${ }^{4}$ Hull Medical School, The University of \\ Hull, Hull, UK. ${ }^{5}$ Amsterdam Institute of Social Science Research, University of Amsterdam, Amsterdam, Netherlands. ${ }^{6}$ Department \\ of Palliative Care, Policy and Rehabilitation, Cicely Saunders Institute, King's College London, London, UK. ${ }^{7}$ Department of Palliative \\ Care, Cambridge University Hospitals, Cambridge, UK
}

Contact address: Anna Bolzani, Department of Palliative Medicine, Munich University Hospital, LMU Munich, Marchioninistr. 15, Munich, Germany. Anna.Bolzani@med.uni-muenchen.de.

Editorial group: Cochrane Pain, Palliative and Supportive Care Group.

Publication status and date: New, published in Issue 6, 2017.

Citation: Bolzani A, Rolser SM, Kalies H, Maddocks M, Rehfuess E, Hutchinson A, Gysels M, Higginson IJ, Booth S, Bausewein C. Cognitive-emotional interventions for breathlessness in adults with advanced diseases. Cochrane Database of Systematic Reviews 2017 , Issue 6. Art. No.: CD012682. DOI: 10.1002/14651858.CD012682.

Copyright (C) 2017 The Cochrane Collaboration. Published by John Wiley \& Sons, Ltd.

\begin{abstract}
A B S T R A C T
This is a protocol for a Cochrane Review (Intervention). The objectives are as follows:

To assess the effects and safety of interventions targeting cognition, emotion or both as the predominant underlying mechanism of effect to relieve breathlessness in adults suffering from advanced diseases.
\end{abstract}

\section{BACKGROUN D}

This protocol is partly based on suggested wording from the Cochrane Pain, Palliative and Supportive Care Review Group $(\mathrm{PaPaS} \mathrm{CRG})$. Some wording is used from the original review (Bausewein 2008), which this new review will update and replace.

\section{Description of the condition}

Breathlessness or dyspnoea is defined as "subjective experience of breathing discomfort that consists of qualitatively distinct sensations that vary in intensity" (Meek 1999). The term 'breathlessness' reflects the patients' perspective based on the daily experi- ence whereas the medical term 'dyspnoea' focuses more on the clinical sign of an underlying condition (Johnson 2014). "The experience derives from interactions among multiple physiological, psychological, social, and environmental factors, and may include secondary physiological and behavioural responses" (Meek 1999). Since this definition was adopted, new evidence has led to better understanding of the mainly sensory and affective components and that dyspnoea "must generally be distinguished from signs that clinicians typically invoke as evidence of respiratory distress, such as tachypn(o)ea, use of accessory muscles, and intercostal retractions." (Parshall 2012). Many patients with different conditions including primary and secondary cancer, lung diseases (e.g. 
chronic obstructive pulmonary disease (COPD), pulmonary hypertension, cystic fibrosis, interstitial lung disease (ILD)), chronic heart failure (CHF) or motor neuron disease/amyotrophic lateral sclerosis (MND/ALS) suffer from this distressing symptom (Bailey 2010; Booth 2008; Breaden 2011; Lansing 2009; Solano 2006). Breathlessness is a multifactorial and complex symptom and an experience unique to the individual (Booth 2008). It is often expressed as air hunger, work of breathing, laboured breathing, awareness of respiratory distress, and shortness of breath or chest tightness (Barnes 2016; Parshall 2012). Breathing discomfort is described by such phrases as 'could not breathe fast or deep enough' or 'could not get enough air' or 'suffocating' (Guz 1997). Breathlessness is one of the most prevalent and distressing symptoms in advanced stages of malignant and non-malignant diseases. Up to $95 \%$ of patients with advanced chronic pulmonary disease, $88 \%$ with advanced heart disease, and $70 \%$ with end stage cancer experience breathlessness in their last year of life (Graham 2010; Lansing 2009; Moens 2014; Solano 2006; Teunissen 2007). The frequency and severity of breathlessness increase during the course of the disease until death (Bailey 2010; Breaden 2011). It is an extremely distressing symptom for the patient but also for the accompanying family and professional carers (Booth 2008).

Overall, breathlessness is still difficult to palliate.

\section{Description of the intervention}

\section{Management of breathlessness}

Appropriate management to relieve breathlessness in advanced diseases requires both pharmacological and non-pharmacological interventions. Different systematic reviews and meta-analyses were published in recent years and analysed the effects of pharmacological interventions such as opioids (Barnes 2016; Mahler 2013), benzodiazepines (Simon 2016), and oxygen (Ameer 2014; Cranston 2008; Sharp 2016) for breathlessness in adult patients.

However, the use of drugs to treat breathlessness is sometimes limited as they entail adverse effects and doses need to be titrated carefully. Therefore, non-pharmacological interventions are an important part of the treatment of breathlessness. As mentioned above, many systematic reviews analysed the effects of pharmacological treatments, which is why we are focusing solely on non-pharmacological interventions in this review.

\section{Non-pharmacological interventions}

Many non-pharmacological interventions for the relief of breathlessness have been developed and evaluated in recent years. For better clarity, we therefore categorise the interventions based on a theoretical concept developed by Booth 2014, Chin 2016 and Spathis 2017. This concept builds on the effect breathlessness has on patients (Figure 1).

Figure I. Perpetuation of breathlessness by vicious cycles (Booth 2014)

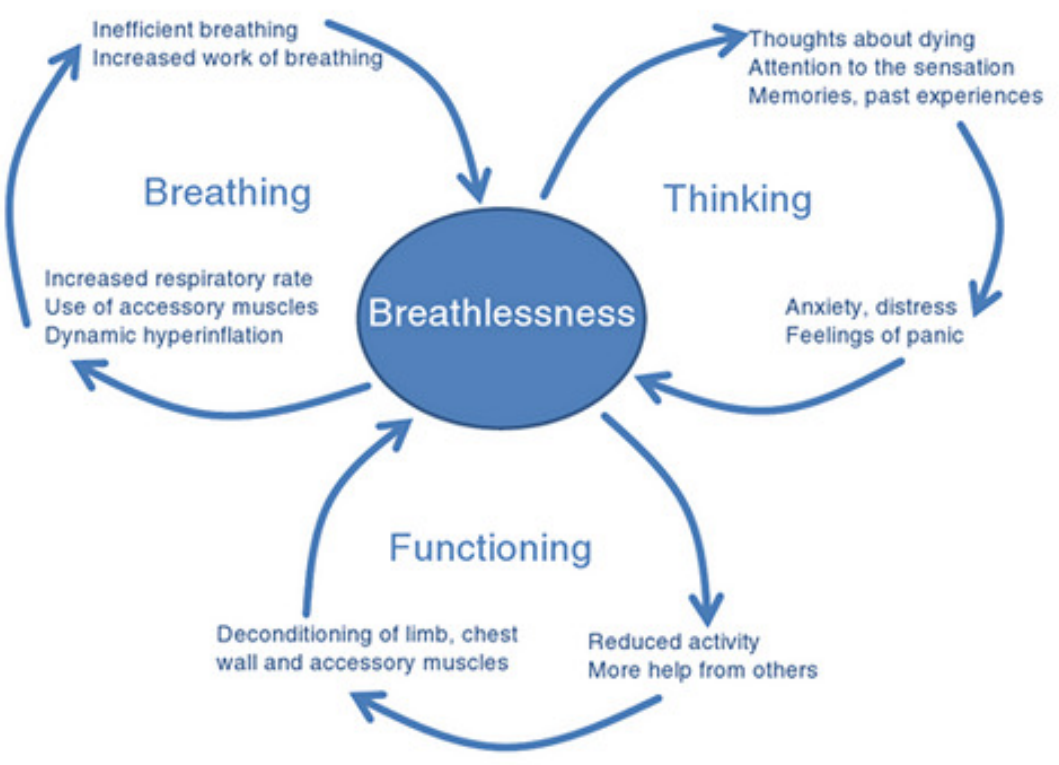

Cognitive-emotional interventions for breathlessness in adults with advanced diseases (Protocol) 
- Respiratory: Inefficient breathing and increased work of breathing can be observed due to dysfunctional breathing patterns with an increased respiratory rate, the need for the use of accessory muscles, and dynamic hyperinflation.

- Cognitive-emotional: Misconceptions and paying too much attention to the sensation of breathlessness such as memories of past or negative experiences lead to anxiety, distress, feelings of panic, and thoughts about dying.

- Physical: Persons suffering from severe breathlessness show reduced physical activity with a tendency to self-isolation and the need for more help from others. This leads to deconditioning of limb, chest wall and accessory muscles.

We expect a huge number of studies and categories of interventions to be included. Therefore, three different reviews, based on the theoretical concept, will be conducted. An additional review is planned, focusing on interventions targeting more than one underlying mechanism as described above.

In this review, we will analyse non-pharmacological interventions targeting primarily cognition and/or emotion to relieve breathlessness in patients suffering from advanced stages of disease, for example distractive auditory stimuli (music), meditation/relaxation (e.g. visual or guided imagery; progressive muscle relaxation), biofeedback, mindfulness-based stress reduction, and psychological therapy (e.g. cognitive behavioural therapy). These interventions may take place in a variety of settings, and can, with guidance of healthcare professionals, mostly be carried out by patients themselves (Figure 2).

Figure 2. System-based logic model on cognitive-emotional interventions for breathlessness in patients with advanced diseases

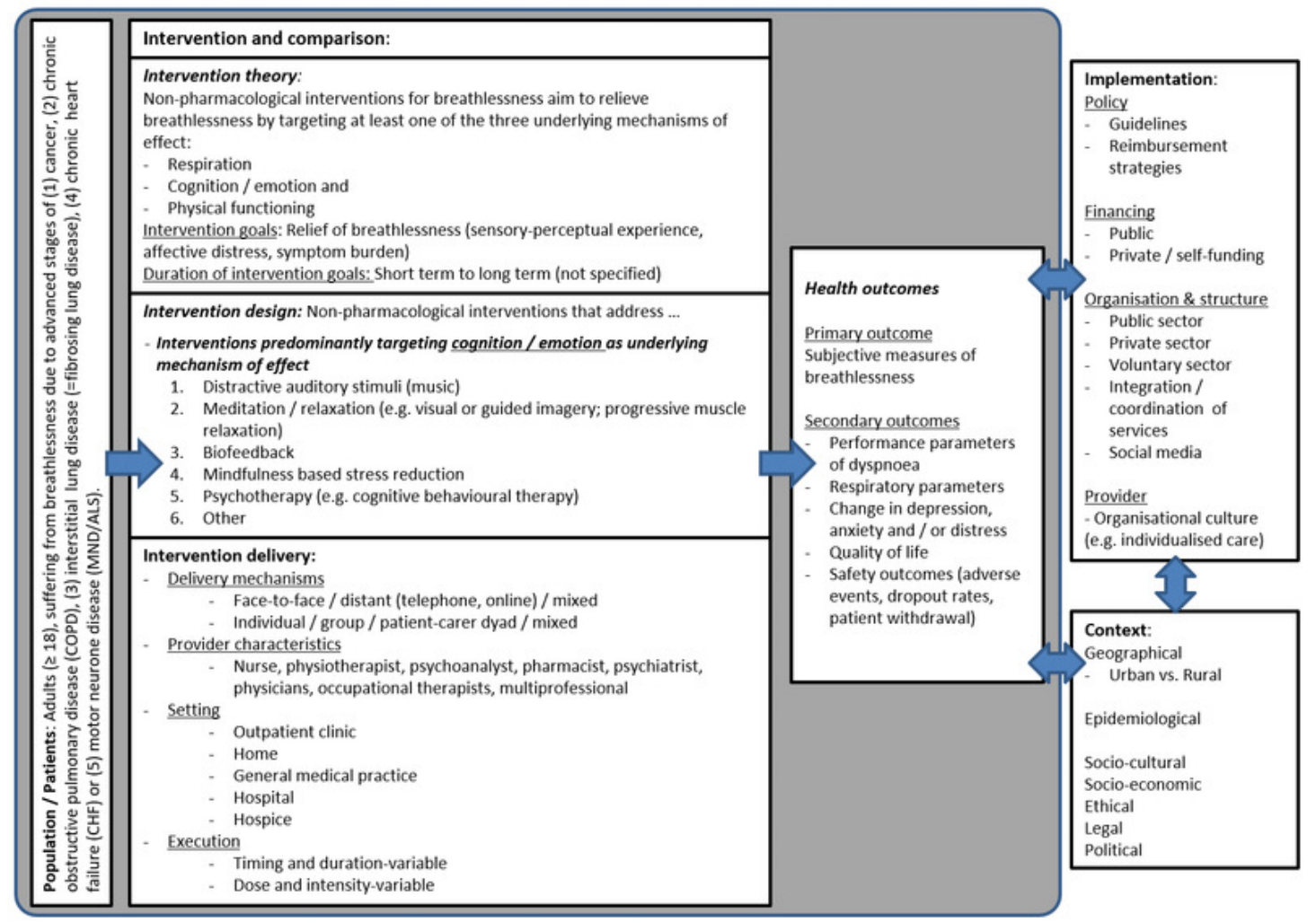

Invasive interventions could also be classified as non-pharmacological but they will not be the focus in this review. Therefore, we will exclude surgical procedures such as drainage, tapping, endoscopy, ventilation and catheterisation.
We will also exclude the following non-pharmacological interventions as there have been recent Cochrane Reviews: pulmonary rehabilitation (McCarthy 2015), and nutrition (Ferreira 2012). 


\section{How the intervention might work}

All interventions that will be subsumed in this review aim to address and modify behaviour, mood, emotional state and cognition. This may help people suffering from advanced diseases to better cope with their breathlessness. Cognitive-emotional interventions target the training of coping strategies, the reduction of fear and stress relating to breathlessness or the enhancement of positive thinking. Different types of cognitive-emotional techniques are of interest in this review.

Psychological therapy, for example cognitive behavioural therapy, aims to help patients to break through the vicious cycle of breathlessness and activity avoidance, by providing, among others, behavioural activation, and problem-solving techniques (von Leupoldt 2012).

Relaxation and meditative techniques aim to produce a stage of relaxation and stress relief through elimination of environmental distractions (Glanze 2012).

Distractive auditory stimuli (music) with and without exercise are supposed to reduce the intensity of symptoms, e.g. breathlessness, through drawing the attention away from the symptoms (Lee 2015).

Based on a template by Rohwer 2017 we developed a system-based logic model in which we show how non-pharmacological interventions for breathlessness, with a focus on interventions predominantly targeting cognition and/or emotion, are implemented in the healthcare system (Figure 2).

\section{Why it is important to do this review}

Non-pharmacological interventions can complement pharmacological interventions and may offer alternative treatment options in the management of breathlessness occurring in advanced illness. As research into this challenging, poorly managed and burdensome symptom is rapidly evolving, there is a need to synthesise the most recent evidence to inform practice and research. Our review aim is to aid health professionals in the treatment of breathlessness with palliative intent and to inform patients and carers about the evidence of non-pharmacological interventions targeting cognition, emotion or both to relieve breathlessness.

This is an update of a Cochrane review on non-pharmacological interventions for the relief of breathlessness in advanced disease (Bausewein 2008). The former review showed effectiveness of neuromuscular electrical stimulation, chest wall vibration, walking aids, and breathing training. The review included 47 studies that were categorised in different intervention groups (e.g. walking aids, acupuncture, breathing training, psychological therapy). Since its publication, many randomised controlled studies on non-pharmacological interventions have been published, including new intervention groups (e.g. breathlessness services). Therefore, although necessary, a single review as an update of the earlier review seemed infeasible. Based on the interventions used to target breathlessness, we decided to assess the interventions in different reviews.

\section{O B J E C T I VES}

To assess the effects and safety of interventions targeting cognition, emotion or both as the predominant underlying mechanism of effect to relieve breathlessness in adults suffering from advanced diseases.

\section{METHODS}

\section{Criteria for considering studies for this review}

\section{Types of studies}

We will include randomised controlled trials (RCTs), cluster RCTs, and quasi-RCTs (QRCTs). Quasi-randomisation is defined as some pseudo-random method of allocation such as alternation, date of birth, case record number or date of presentation (Higgins 2011). We will include cross-over studies, if separate data for both time periods are presented. We will only use the data of the first period for analysis to avoid carry-over effects. We will require full journal publication. Where full journal publication is not available, we will try to obtain data by contacting the trial authors unless sufficient data for analyses are provided in online clinical trial results, summaries of otherwise unpublished clinical trials, or conference abstracts. QRCTs will be included in order to obtain the full breadth of relevant trials, in particular as we expect to find a small number of RCTs for some of the intervention categories; we are aware of the higher risk of bias in these studies and will account for this in the analysis.

\section{Types of participants}

Adult patients aged 18 years and above, suffering from advanced diseases with a high prevalence of breathlessness.

We will include studies if the majority ( $\geq 50 \%$ ) of participants meet the following criteria.

- Patients suffering from cancer should have advanced local or metastatic disease (e.g. TNM Classification of Malignant Tumours (TNM) state $\geq \mathrm{T} 3$ or $\mathrm{N} \geq 1$ or $\mathrm{M} \geq 1$ ).

- Patients with severe COPD should have a forced expiratory volume in one second (FEV1) predicted of $<50 \%$.

- Patients with pulmonary hypertension will be included if they reach a WHO class level $\geq$ III, defined by Barst 2004 .

- Patients suffering from CHF should have New York Heart Association (NYHA) stage III or IV.

- Patients with ILD or idiopathic pulmonary fibrosis (IPF) : all studies will be included as breathlessness is the predominant 
symptom and there are hardly any disease-specific treatment options.

- Patients with neuromuscular diseases (MND, ALS): all studies will be included as advanced disease is marked by the occurrence of breathlessness.

If groups for the inclusion criteria mentioned above were stratified, we will only include the subgroups of interest. We will document difficult decisions in the review. Sensitivity analysis can assess the impact of these decisions on the review's result. Patients included in the studies can be in any setting. We will exclude studies of patients with any condition not regarded as advanced and lifelimiting such as acute or chronic asthma, or with pre-existing diagnosis of acute asthma or acute cardiac condition as a primary cause of breathlessness.

\section{Types of interventions}

We will include interventions targeting cognition, emotion or both to relieve breathlessness according to the following prespecified categories.

- Distractive auditory stimuli (music).

- Meditation/relaxation (e.g. visual/guided imagery;

progressive muscle relaxation).

- Biofeedback.

- Mindfulness-based stress reduction.

- Psychological therapy (e.g. cognitive behavioural therapy).

If we find interventions of interest that do not fit in the above categories, we will define an additional category 'Other' or add new categories if there is a sufficient number of studies.

The judgement for inclusion will be based on the study authors' description of the intervention; any deviation from this will be explicitly mentioned.

Interventions may take place in any setting, e.g. outpatient clinic, home, hospital, hospice, general medical practice.

The comparator may be no treatment, placebo, attention control, standard care, or a different kind of therapy. We will categorise the control groups into 'active controls' or 'other' based on the description of the comparison group. We will focus on active controls as comparison group in our primary analysis. Concomitant interventions, especially pharmacological treatment, will be accepted, if administered in the same way in both the control and the treatment groups. If these interventions are suspected to have some relevant influence on our outcomes we will consider this in subgroup analysis.

\section{Types of outcome measures}

We anticipate that studies will use a variety of outcome measures. To be included, a study must have any measure of breathlessness. Adverse effects of cognitive-emotional interventions will be measured as absent or present and a narrative description of these ef- fects will be given when reported. We will consider all reliable and validated measures for the following outcomes.

\section{Primary outcomes}

Breathlessness, measured by self-reported instruments with a focus on breathlessness or mastery of breathlessness (e.g. Baseline Dyspnoea Index (BDI), Borg Dyspnoea Scale (BDS), Medical Research Council (MRC) Breathlessness Scale, or Chronic Respiratory Disease Questionnaire (CRQ)). Other terms for breathlessness such as dyspnoea, shortness of breath, and difficulty breathing will also be accepted.

\section{Secondary outcomes}

- Performance parameters (e.g. walking tests, International Physical Activity Questionnaire (IPAQ)).

- Respiratory parameters (e.g. change in FEV1(\%)).

- Change in depression, anxiety and/or distress (e.g. Hospital Anxiety and Depression Scale (HADS)).

- Quality of life (e.g. 36-Item Short Form Health Survey (SF36)).

- Safety outcomes:

- Adverse events (measured as absent or present);

- Dropout rates; and

- Patient withdrawal from the trial, due to any reason (if mentioned).

\section{Search methods for identification of studies}

\section{Electronic searches}

We will search the following databases from their inception to the present, without date or language restrictions.

- Cochrane Database of Systematic Reviews (CDSR), the Cochrane Library.

- Cochrane Central Register of Controlled Trials

(CENTRAL), the Cochrane Library.

- MEDLINE (Ovid).

- Embase (Ovid).

- PsycINFO (Ovid).

- LILACS (Bireme)

- CINAHL (Ebsco).

We will search MEDLINE and Embase using both controlled vocabulary (namely, MeSH in MEDLINE and EMTREE in Embase) and a wide range of free-text terms. To detect all RCTs we will perform the search on MEDLINE using the Cochrane Highly Sensitive Search Strategy, sensitivity-maximising version (Higgins 2011).

The search strategy for MEDLINE is in Appendix 1. 


\section{Searching other resources}

We will search the meta-register of controlled trials (mRCT) (www.controlled-trials.com/mrct), clinicaltrials.gov ( www.clinicaltrials.gov) and the WHO International Clinical Trials Registry Platform (ICTRP) (apps.who.int/trialsearch/) for ongoing trials. In addition, we will check reference lists of reviews and retrieved articles for additional studies, and we will perform citation searches on key articles. We will contact experts in the field for unpublished and ongoing trials. We will contact study authors where necessary for additional information.

We will perform the search in collaboration with the Information Specialist of the Cochrane Pain, Palliative and Supportive Care Group.

\section{Data collection and analysis}

\section{Selection of studies}

Two review authors (AB, SR) will independently screen all titles and abstracts retrieved by the search to identify all trials that may be eligible and for which the full paper should be obtained. Independent review authors will eliminate studies that clearly do not satisfy inclusion criteria, and obtain full copies of the remaining studies. Two review authors (AB, SR) will read these studies independently to select relevant studies, and in the event of disagreement or unclear decision to include, we will resolve disagreement with a third author (MM or CB, depending on the topic). We will not anonymise the studies in any way before assessment.

We will include a PRISMA flow chart in the full review which will show the status of identified studies (Moher 2009) as recommended in Part 2, Section 11.2.1 of the Cochrane Handbook for Systematic Reviews of Interventions (Higgins 2011). We will include studies in the review irrespective of whether measured outcome data are reported in a 'usable' way.

\section{Data extraction and management}

Two review authors ( $\mathrm{SR}, \mathrm{AB}, \mathrm{AH}$ or $\mathrm{MM}$ ) will independently extract data using a data collection form based on a standard form released by the Cochrane Effective Practice and Organisation of Care Group (EPOC) and check for agreement before entry into Review Manager (RevMan 2014). Where there is disagreement, a third author (CB or SB) will be consulted to resolve differences. We will include information about the following.

\section{Participant characteristics}

- Demographic characteristics (age, gender, nationality).

- Underlying disease characteristics (type and stage of condition).

\section{Intervention}

- Intervention theory

- Type of intervention (description of intervention, frequency, duration (total and per session)).

- Types of control condition (control intervention, control group).

- Type of delivery (delivery mechanisms such as face-to-face, distant; group, individual; provider characteristics such as nurses, physicians, multiprofessional; setting such as outpatient clinic, home, hospital).

\section{Methods}

- Study design.

- Size of intervention and control group at baseline and follow-up.

- Study duration and follow-up.

- Sources of bias (sequence generation, allocation sequence concealment, blinding, incomplete outcome data, selective reporting, other concerns about bias).

\section{Outcomes}

- Key outcomes with measurement instruments.

- Timing, duration and frequency of follow-up.

- Adverse events.

- Number of withdrawals and dropouts.

\section{Context}

- Country of origin.

In case multiple reports of the same study are found, we will extract data of all these reports independently of each other and compare; if data differ between reports, all authors will make a decision how to treat this study and this will be documented in the review. We will collate multiple reports of the same study, so that each study rather than each report is the unit of interest in the review. We will collect characteristics of the included studies in sufficient detail to populate a table of 'Characteristics of included studies' in the full review. Review authors will not be involved in the data extraction of studies they authored or co-authored.

\section{Assessment of risk of bias in included studies}

Two authors $(\mathrm{AB}, \mathrm{AH})$ will independently assess risk of bias for each study, using the criteria outlined in the Cochrane Handbook for Systematic Reviews of Interventions (Higgins 2011) and adapted from those used by the Cochrane Pregnancy and Childbirth Group.

We will assess the following for each study.

- Random sequence generation (checking for possible selection bias). We will assess the method used to generate the 
allocation sequence as: low risk of bias (any truly random process, e.g. random number table; computer random number generator); unclear risk of bias (method used to generate sequence not clearly stated). Studies using a non-random process (e.g. odd or even date of birth; hospital or clinic record number) will be assessed as high risk of bias.

- Allocation concealment (checking for possible selection bias). The method used to conceal allocation to interventions prior to assignment determines whether intervention allocation could have been foreseen in advance of, or during, recruitment, or changed after assignment. We will assess the methods as: low risk of bias (e.g. telephone or central randomisation; consecutively numbered sealed opaque envelopes); high risk of bias (studies that do not conceal allocation (e.g. open list); unclear risk of bias (method not clearly stated).

- Blinding of outcome assessment (checking for possible detection bias). We will assess the methods used to blind study participants and outcome assessors from knowledge of which intervention a participant received. We will assess the methods as: low risk of bias (study states that it was blinded and describes the method used to achieve blinding); high risk of bias (no or incomplete blinding); unclear risk of bias (study states that it was blinded but does not provide an adequate description of how it was achieved). We will also report if study participants are asked about their expectations of benefit of intervention/control if blinding is not feasible.

- Incomplete outcome data (checking for possible attrition bias due to the amount, nature and handling of incomplete outcome data). We will assess the methods used to deal with incomplete data as: low risk $(<10 \%$ of participants did not complete the study and/or data have been imputed using appropriate methods); high risk of bias (used 'completer' analysis); unclear risk of bias (insufficient information for low/ high risk of bias category).

- Selective reporting (checking for reporting bias). We will assess the methods as: low risk of bias (where it is clear that all of the study's prespecified outcomes and all expected outcomes of interest to the review have been reported); high risk of bias (where not all the study's prespecified outcomes have been reported; one or more reported primary outcomes were not prespecified; outcomes of interest are reported incompletely and so cannot be used; study fails to include results of a key outcome that would have been expected to have been reported); unclear risk of bias (insufficient information for low/high risk of bias category).

- Other bias (e.g. checking for possible biases confounded by small size. We will assess studies as being at low risk of bias $(\geq$ 200 participants per treatment arm); unclear risk of bias (50 to 199 participants per treatment arm); high risk of bias $(<50$ participants per treatment arm)).

We will use the Review Manager tool to complete a 'Risk of bias' table (RevMan 2014). Any discrepancy between the two authors will be resolved by discussion involving a third author (CB).

\section{Measures of treatment effect}

We will analyse dichotomous outcomes using risk ratios (RRs) with $95 \%$ confidence intervals (CIs). We will recategorise any categorical outcomes with more than two categories into two groups. We will analyse continuous data using standardised mean differences (SMDs) with 95\% CIs. We will calculate standard deviations, if not reported, using the methods described in the Cochrane Handbook for Systematic Reviews of Interventions (Higgins 2011). We plan to report the proportion of participants experiencing any adverse effects of cognitive-emotional interventions, and combine studies using RRs with 95\% CIs.

\section{Unit of analysis issues}

We will reanalyse data, if possible, for cluster trials which have not taken clustering into account in their analysis. We will calculate effective sample sizes and adjusted standard errors using the design effect method. We will try to obtain estimates for intracluster correlation coefficients from study authors or will use external estimates obtained from comparable studies, as recommended by Cochrane guidelines (Higgins 2011). We will document if reanalysis is not feasible.

In studies with more than two arms, we will consistently choose the active control arms in the main analysis, and, if possible, do a sensitivity analysis, in which we will choose the other control arm. We will combine individually randomised controlled trials and cluster RCTs in the same meta-analyses or harvest plots, but these will be clearly identified (Higgins 2011).

\section{Dealing with missing data}

We will contact study authors if missing data on study characteristics or outcome measures precludes study inclusion or limits use of a study at further stages of the review. If studies do not report outcomes based on intention-to-treat analyses this will be considered as a source of bias during 'Risk of bias' assessment. We will try to calculate effect measures or CIs wherever possible from available data, if we get no response.

\section{Assessment of heterogeneity}

We will assess methodological and clinical heterogeneity with tables documenting the following characteristics of the included studies.

- Intervention components (e.g. music, meditation/ relaxation, biofeedback, psychological therapy).

- Intervention delivery mechanism (e.g. face-to-face, distant).

- Provider characteristics (e.g. nurses, physiotherapists, physicians).

- Setting (e.g. outpatient clinic, hospice, home). 
- Patients (e.g. COPD, cancer, fibrosing lung disease).

- Methods (outcome measures, outcome assessment).

For those studies assessing the impacts of a given intervention category on comparable outcomes, thus making pooling through meta-analysis feasible, we will assess statistical heterogeneity graphically with a forest plot by examining the extent to which CIs overlap, and statistically with the $\mathrm{I}^{2}$ statistic. We will consider an $\mathrm{I}^{2}$ value greater than $50 \%$ to indicate substantial statistical heterogeneity, and will consider it statistically significant if the $\mathrm{P}$ value for the $\mathrm{Chi}^{2}$ test is $<0.1$. We will document statistical heterogeneity but this will not have any direct consequences for meta-analysis (see below). We will create forest plots and $\mathrm{I}^{2}$ calculations using Review Manager 5.3 (RevMan 2014).

\section{Assessment of reporting biases}

We will try to minimise publication bias by searching trials registers for projected and registered studies that have never been published. We will contact the authors to get unpublished information if there are such studies registered or some relevant information is missing and can therefore narrow the risk of reporting bias. We will assess the possibility that publication bias affects the review using funnel plots when at least 10 studies are available for metaanalysis.

\section{Data synthesis}

We will attempt to pool all studies within a given intervention category assessing the same outcome by conducting a meta-analysis using Review Manager 5.3 (RevMan 2014). We will use the random-effects model due to the expected large heterogeneity in delivery mechanisms, provider characteristics, setting and study population.

We will report results as RRs for dichotomous outcomes and SMDs for continuous outcomes. We will undertake meta-analysis only if studies are judged to be similar enough to give a clinically meaningful answer. We will provide an outcome table and summarise the results narratively if meta-analysis is not possible.

In the case of skewed data, we will log transform these data for our analysis or, if that approach is not feasible, summarise them narratively.

\section{'Summary of findings' table}

We will include a 'Summary of findings' table using the GRADE profiler software (GRADEpro GDT 2015) as set out in the PaPaS author guide (AUREF 2012) and recommended in the Cochrane Handbook for Systematic Reviews of Interventions, Chapter 4.6.6 (Higgins 2011) to evaluate the quality of evidence in our review. The 'Summary of findings' table will include outcomes of: a) change of breathlessness, b) objective parameters of breathlessness, c) quality of life indicators, d) change of depression or anxiety, e) adverse events, f) characteristics of the patient population that benefits most.

\section{Quality of the evidence}

This section is taken from the Cochrane Drugs and Alcohol Group recommended text. The overall quality of the evidence for each outcome in our review will be assessed using the GRADE system (GRADEpro GDT 2015) and presented in the 'Summary of findings' tables, to present the main findings of a review in a transparent and simple tabular format. In particular, we will include key information concerning the quality of evidence, the magnitude of effect of the interventions examined, and the sum of available data on the main outcomes.

The GRADE system uses the following criteria for assigning grade of evidence.

- High: we are very confident that the true effect lies close to that of the estimate of the effect.

- Moderate: we are moderately confident in the effect estimate; the true effect is likely to be close to the estimate of effect, but there is a possibility that it is substantially different.

- Low: our confidence in the effect estimate is limited; the true effect may be substantially different from the estimate of the effect.

- Very low: we have very little confidence in the effect estimate; the true effect is likely to be substantially different from the estimate of effect.

We will decrease grade rating by one (-1) or two (-2) if we identify:

- serious (-1) or very serious (-2) limitation to study quality;

- important inconsistency $(-1)$;

- some (-1) or major (-2) uncertainty about directness;

- imprecise or sparse data $(-1)$; or

- high probability of reporting bias (-1).

\section{Subgroup analysis and investigation of heterogeneity}

We will undertake subgroup analysis for the primary outcomes to examine factors that may explain variation in the effectiveness, if numbers are sufficiently large. We will perform stratification as follows.

- Type of intervention.

- Intervention delivery (delivery mechanisms such as face-toface, distant; group, individual; provider characteristics such as nurses, physicians, multiprofessional; setting such as outpatient clinic, home, hospital).

- Patient characteristics (underlying disease, disease stage, age, gender).

- Underlying therapy

\section{Sensitivity analysis}


We will conduct sensitivity analysis where possible, to test the effect of different methodological decisions made throughout the review process on the primary outcome. We will test the robustness of the results by removing from the pooled effect estimate:

- studies with a high risk of bias for two or more key domains;

- quasi-randomised clinical trials;

- outcome measures; and

- intervention of varying duration.

\section{ACKNOWLEDGEMENTS}

Cochrane Review Group funding acknowledgement: this project was supported by the National Institute for Health Research (NIHR), via Cochrane Infrastructure funding to the Cochrane Pain, Palliative and Supportive Care Review Group ( $\mathrm{PaPaS})$. The views and opinions expressed herein are those of the authors and do not necessarily reflect those of the Systematic Reviews Programme, NIHR, National Health Service (NHS) or the Department of Health.

We are grateful to Amanda C de C Williams and Chris Eccleston for providing helpful comments on the concept and earlier drafts of the protocol. The support of Joanne Abbott (Information Specialist for $\mathrm{PaPaS}$ ) in developing the search strategy is gratefully acknowledged.

\section{R E F E R E N C E S}

\section{Additional references}

\section{Ameer 2014}

Ameer F, Carson KV, Usmani ZA, Smith BJ. Ambulatory oxygen for people with chronic obstructive pulmonary disease who are not hypoxaemic at rest. Cochrane Database of Systematic Reviews 2014, Issue 6. [DOI: 10.1002/ 14651858.CD000238.pub2]

\section{AUREF 2012}

Cochrane Pain, Palliative and Supportive Care Group. $\mathrm{PaPaS}$ author and referee guidance. papas.cochrane.org/ papas-documents (accessed 6 July 2016).

Bailey 2010

Bailey CD, Wagland R, Dabbour R, Caress A, Smith J, Molassiotis A. An integrative review of systematic reviews related to the management of breathlessness in respiratory illnesses. BMC Pulmonary Medicine 2010;10(63):1-13. [DOI: 10.1186/1471-2466-10-63]

Barnes 2016

Barnes H, McDonald J, Smallwood N, Manser R. Opioids for the palliation of refractory breathlessness in adults with advanced disease and terminal illness. Cochrane Database of Systematic Reviews 2016, Issue 3. [DOI: 10.1002/ 14651858.CD011008.pub2]

Barst 2004

Barst RJ, McGoon M, Torbicki A, Sitbon O, Krowka MJ, Olschewski H, et al. Diagnosis and differential assessment of pulmonary arterial hypertension. Journal of the American College of Cardiology 2004;43(12):40-7. [DOI: 10.1016/ j.jacc.2004.02.032]

Booth 2008

Booth S, Moosavi SH, Higginson IJ. The etiology and management of intractable breathlessness in patients with advanced cancer: a systematic review of pharmacological therapy. Nature Clinical Practice. Oncology 2008;5(2): 90-100. [DOI: 10.1038/ncponc1034]

\section{Booth 2014}

Booth S, Burkin J, Moffat C, Spathis A. Managing Breathlessness in Clinical Practice. London: Springer-Verlag, 2014:396-8. [DOI: 10.1007/978-1-4471-4754-1; ISBN 978-1-4471-4754-1]

Breaden 2011

Breaden K. Recent advances in the management of breathlessness. Indian Journal of Palliative Care 2011;17: 29-32. [PUBMED: 3140084]

\section{Chin 2016}

Chin C, Booth S. Managing breathlessness: a palliative care approach. Postgraduate Medical Journal April 2016;92: 393-400. [DOI: 10.1136/postgradmedj-2015-133578]

\section{Cranston 2008}

Cranston JM, Crockett A, Currow D. Oxygen therapy for dyspnoea in adults. Cochrane Database of Systematic Reviews 2008, Issue 3. [DOI: 10.1002/ 14651858.CD004769.pub2]

\section{Ferreira 2012}

Ferreira IM, Brooks D, White J, Goldstein R. Nutritional supplementation for stable chronic obstructive pulmonary disease. Cochrane Database of Systematic Reviews 2012, Issue 12. [DOI: 10.1002/14651858.CD000998.pub3]

Glanze 2012 Glanze WD, Anderson K, Anderson LE. Mosby's Medical, Nursing, and Allied Health Dictionary. 9th Edition. Elsevier/ Mosby, 2012. [ISBN-10: 0323074030]

GRADEpro GDT 2015 [Computer program] GRADE Working Group, McMaster University. GRADEpro GDT. Version accessed 01 July 2016. Hamilton (ON): GRADE Working Group, McMaster University, 2015.

\section{Graham 2010}

Graham L. ACCP releases statement on dyspnea treatment in patients with advanced lung or heart disease. American Family Physician 2010;82(8):999-1000. 
Guz 1997

Guz A. Brain, breathing and breathlessness. Respiration Physiology 1997;109(3):197-204.

Higgins 2011

Higgins JPT, Green S (editors). Cochrane Handbook for Systematic Reviews of Interventions Version 5.1.0 (updated March 2011). The Cochrane Collaboration, 2011. Available from handbook.cochrane.org. Available from www.cochrane-handbook.org. The Cochrane Collaboration

\section{Johnson 2014}

Johnson MJ, Currow DC, Booth S. Prevalence and assessment of breathlessness in the clinical setting. Expert Review of Respiratory Medicine 2014;8(2):151-61. [DOI: 10.1586/17476348.2014.879530]

\section{Lansing 2009}

Lansing RW, Gracely RH, Banzett RB. The multiple dimensions of dyspnea: review and hypotheses. Respiratory Physiology \& Neurobiology 2009;167(1):53-60. [DOI: 10.1016/j.resp.2008.07.012]

Lee 2015

Lee AL, Desveaux L, Goldstein RS, Brooks D. Distractive auditory stimuli in the form of music in individuals with COPD: a systematic review. CHEST Journal 2015;148: 417-29. [DOI: 10.1378/chest.14-2168]

\section{Mahler 2013}

Mahler DA. Opioids for refractory dyspnea. Expert Review of Respiratory Medicine 2013;7(2):123-35. [DOI: 10.1586/ ers.13.5]

McCarthy 2015

McCarthy B, Casey D, Devane D, Murphy K, Murphy

E, Lacasse Y. Pulmonary rehabilitation for chronic obstructive pulmonary disease. Cochrane Database of Systematic Reviews 2015, Issue 2. [DOI: 10.1002/ 14651858.CD003793.pub3]

\section{Meek 1999}

Meek PM, Schwartzstein RM, Adams L, Altose MD, Breslin EH, Carrieri-Kohlman V, et al. Dyspnea mechanisms, assessment, and management: a consensus statement. American Journal of Respiratory and Critical Care Medicine 1999;159(1):321-40. [DOI: 10.1164/ajrccm.159.1.ats898]

\section{Moens 2014}

Moens K, Higginson IJ, Harding R. Are there differences in the prevalence of palliative care-related problems in people living with advanced cancer and eight noncancer conditions? A systematic review. Journal of Pain and Symptom Management 2014;48(4):660-77. [DOI: 10.1016/j.jpainsymman.2013.11.009]

Moher 2009

Moher D, Liberati A, Tetzlaff J, Altman DG, the PRISMA Group. Preferred reporting items for systematic reviews and meta-analyses: the PRISMA statement. PLoS Medicine 2009;6(7):e1000097.
Parshall 2012

Parshall MB, Schwartzstein RM, Adams L, Banzett RB, Manning HL, Bourbeau J, et al. An official American Thoracic Society Statement: update on the mechanisms, assessment, and management of dyspnea. American Journal of Respiratory and Critical Care Medicine 2012;185(4):43552. [DOI: $10.1164 / \mathrm{rccm} .201111-2042 \mathrm{ST}$ ]

RevMan 2014 [Computer program] Nordic Cochrane Centre, The Cochrane Collaboration. Review Manager. Version 5.3. Copenhagen: Nordic Cochrane Centre, The Cochrane Collaboration, 2014.

\section{Rohwer 2017}

Rohwer A, Booth A, Pfadenhauer L, Burns J, Brereton L, Gerhardus A, et al. Logic models help make sense of complexity in systematic reviews and health technology assessments. Journal of Clinical Epidemiology 2017;83: $37-47$.

Sharp 2016

Sharp C, Adamali H, Millar AB. Ambulatory and shortburst oxygen for interstitial lung disease. Cochrane Database of Systematic Reviews 2016, Issue 7. [DOI: 10.1002/ 14651858.CD011716.pub2]

Simon 2016

Simon ST, Higginson IJ, Booth S, Harding R, Weingärtner V, Bausewein C. Benzodiazepines for the relief of breathlessness in advanced malignant and nonmalignant diseases in adults. Cochrane Database of Systematic Reviews 2016, Issue 10. [DOI: 10.1002/ 14651858.CD007354.pub3]

\section{Solano 2006}

Solano JP, Gomes B, Higginson IJ. A comparison of symptom prevalence in far advanced cancer, AIDS, heart disease, chronic obstructive pulmonary disease and renal disease. Journal of Pain and Symptom Management 2006;31 (1):58-69. [DOI: 10.1016/j.jpainsymman.2005.06.007]

\section{Spathis 2017}

Spathis A, Booth S, Moffat C, Hurst R, Ryan R, Chin C, Burkin J. The Breathing, Thinking, Functioning clinical model: a proposal to facilitate evidence-based breathlessness management in chronic respiratory disease. Primary Care Respiratory Medicine 2017;27. [DOI: 10.1038/ s41533-017-0024-z]

Teunissen 2007

Teunissen SC, Wesker W, Kruitwagen C, de Haes HC, Voest EE, de Graeff A. Symptom prevalence in patients with incurable cancer: a systematic review. Journal of Pain and Symptom Management 2007;34(1):94-104. [DOI: 10.1016/j.jpainsymman.2006.10.015]

von Leupoldt 2012 von Leupoldt A, Fritzsche A, Trueba AF, Meuret AE, Ritz T. Behavioral medicine approaches to chronic obstructive pulmonary disease. Annals of Behavioral Medicine 2012;441 (1):52-65. [DOI: 10.1007/s12160-012-9348-7]

References to other published versions of this review 


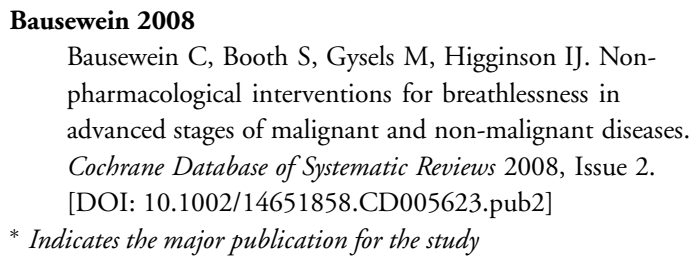

\section{A P P E N D I C E S}

\section{Appendix I. Search strategy for MEDLINE (Ovid)}

1. exp Dyspnea/

2. dyspn?ea.tw.

3. (short* adj2 breath).tw.

4. (urge* adj2 breath*).tw.

5. breathless*.tw.

6. ((labo?red or difficult* or small) adj3 breath $\left.{ }^{*}\right)$.tw.

7. ((respirat* or breath*) adj3 (distress* or comfort $^{*}$ or discomfort $\left.{ }^{*}\right)$ ).tw.

8. (air adj3 (hunger or starve* or need* or gasp* or pant*)).tw.

9. suffocat*.tw.

10. unsatisf* inspiration.tw.

11. or/1-10

12. Neoplasms/ or Lung Neoplasms/

13. ((lung* or bronchi* or pulmo*) adj3 (neoplasm* or cancer* or tumo? ${ }^{*}$ or metasta* or malignan*)).mp.

14. Lung diseases/

15. exp Pulmonary Disease, Chronic Obstructive/

16. (COPD or COAD).tw.

17. Lung Diseases, Obstructive/

18. (obstruct* adj3 (pulmonary or lung* or airway* or airflow* or bronch* or respirat*)).tw.

19. hypertension, pulmonary/

20. or/12-19

21. exp Heart Failure/

22. ((heart or cardia* or myocard*) adj2 (fail* or insufficienc $\left.{ }^{*}\right)$ ).tw.

23. (decompensat* adj2 (heart* or cardia*)).tw.

24. decompensatio cordis.tw.

25. insufficientia cardis.tw.

26. ((cardiac or heart) adj 2 incompetenc*).tw.

27. cardiac stand still.tw.

28. or/21-27

29. exp Lung Diseases, Interstitial/

30. (interstitial adj3 (disease* or pneumoni* or fibrosis)).tw.

31. pulmonary fibrosis.tw.

32. fibrosing alveolitis.tw.

33. Cystic Fibrosis/

34. (cystic fibrosis or mucoviscidosis).tw.

35. or/29-34

36. exp Motor Neuron Disease/

37. (MND or ALS).tw.

Cognitive-emotional interventions for breathlessness in adults with advanced diseases (Protocol)

Copyright @ 2017 The Cochrane Collaboration. Published by John Wiley \& Sons, Ltd. 
38. motor neuron disease*.tw.

39. sclerosis.tw.

40. Amyotrophic Lateral Sclerosis/

41. charcot disease*.tw.

42. lou gehrig disease*.tw.

43. encephalomyelitis disseminate.mp.

44. or/36- 43

45. 20 or 28 or 35 or 44

46. ((end stage or advanc* or final or terminal* or limit*) adj3 (disease* or illness*)).tw.

47. Terminally Ill/

48. Terminal Care/

49. Palliative Care/

50. Prognosis/

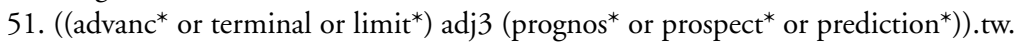

52. disease progression/

53. ((incurable or worsen* ${ }^{*}$ or chronic) adj3 (illness* or disease*)).tw.

54. or/46-53

55.45 or 54

56. randomized controlled trial.pt.

57. controlled clinical trial.pt.

58. randomized.ab.

59. placebo.ab.

60. drug therapy.fs.

61. randomly.ab.

62. trial.ab.

63. groups.ab.

64.56 or 57 or 58 or 59 or 60 or 61 or 62 or 63

65. exp animals/ not humans.sh.

66. 64 not 65

67. 11 and 55

68.66 and 67

\section{CONTRIBUTIONSOFAUTHORS}

Developed concept of review: HK, CB, MM, ER, SB, IJH, MG.

Drafted the protocol: $\mathrm{HK}, \mathrm{AB}, \mathrm{SR}$.

Checked and approved the draft: CB, SB, MM, MG, ER.

Developed search strategy: SR.

\section{DECLARATIONSOF INTEREST}

$\mathrm{AB}$ : none known.

SR: none known.

HK: none known.

MM: none known. MM is investigator on studies that might be included in this review; MM is a specialist physiotherapist and manages patients with breathlessness and advanced disease.

ER: none known.

AH: none known.

Cognitive-emotional interventions for breathlessness in adults with advanced diseases (Protocol)

Copyright @ 2017 The Cochrane Collaboration. Published by John Wiley \& Sons, Ltd. 
MG: none known.

$\mathrm{IJH}$ : none known. IJH is investigator on studies that might be included in this review; $\mathrm{IJH}$ is a specialist physician in palliative medicine and manages patients with breathlessness and advanced disease.

SB: has received payment for talks in Feb 2016 from Novartis. SB is investigator on studies that might be included in this review; SB is a specialist physician in palliative medicine and manages patients with breathlessness and advanced disease.

CB: has received payment for one talk in Oct 2015 from Bayer Health Care. CB is investigator on studies that might be included in this review; $\mathrm{CB}$ is a specialist physician in palliative medicine and manages patients with breathlessness and advanced disease.

\section{SOURCES OF SUPPORT}

\section{Internal sources}

- No sources of support supplied

\section{External sources}

- German Federal Ministry of Education and Research (BMBF), GermanyFunding (Förderkennzeichen 01KG1502), Germany. 